\section{P23 ESSENTIAL BUT PARTIALLY REDUNDANT ROLES FOR POU4F1/BRN-3A AND POU4F2/BRN-3B TRANSCRIPTION FACTORS IN THE DEVELOPING HEART}

L Maskell, K Qamar, TA Hawkins, VS Budhram-Mahadeo. Institute of Cardiovascular Science, UCL Rayne Building, London, UK

\subsection{6/heartjnl-2018-BSCR.28}

Rationale Transcription factors (TFs), which control cardiac gene expression, are important regulators of normal heart development and aberrant expression can contribute to congenital heart defects (CHD), associated with embryonic or neonatal lethality. Brn-3a/POU4F1 and Brn-3b/POU4F2 TFs are expressed in hearts and isolated cardiomyocyte cultures. Studies in injured mouse heart showed that Brn-3b promoted apoptosis if coexpressed with p53 whereas Brn-3a protected against p53 mediated death. Their roles in the developing heart are not known so formed the basis for these studies.

Results Brn-3a and Brn-3b displayed partial compensatory effects in developing mouse hearts because increased Brn-3b in Brn-3a knock-out (KO) hearts supported survival during gestation, whereas double KO mutants (Brn-3a-/-:Brn-3b-/-) showed early embryonic lethality (e9.5). Brn-3a and Brn-3b are highly conserved $(>80 \%)$ between mouse and zebrafish (ZF) so studies with morpholino oligonucleotides $(\mathrm{MO})$ were used to show that reducing both Brn-3a and Brn-3b caused significant cardiac defects (abnormal looping and valve formation) in double morphants, not seen in single or control morphants. However, increased Brn-3b and its target genes e.g. cyclin D1 in Brn-3a KO mutant hearts during mid-gestation correlated with hyperplastic growth in valve/ septum. At later stages, loss of Brn-3a and increased Brn-3b resulted in cardiomyocyte apoptosis, ventricular wall/septal thinning/non-compaction that may contribute to death of Brn-3a KO mutants soon after birth.

Conclusion Our results suggest essential but partially redundant roles for Brn-3a/POU4F1 and Brn-3b/POU4F2 transcription factors (TFs) in the developing heart.

\section{P24 THE FORWARD MYOCARDIAL CREATINE KINASE REACTION IS INCREASED IN OBESITY}

JJ Rayner, W Clarke, MA Peterzan, CT Rodgers, S Neubauer, OJ Rider. OCMR, Divison of Cardiovascular Medicine, University of Oxford

\subsection{6/heartjin-2018-BSCR.29}

Background Obesity is associated with impaired myocardial energetics (reduced phosphocreatine to adenosine triphosphate ratio (PCr/ATP)). However, LV systolic function is usually preserved, suggesting that the rate of ATP delivery to the contractile apparatus may be maintained. We hypothesised that the forward rate constant of the creatine kinase (CK) reaction would increase to compensate for the reduced substrate pool.

Methods 145 participants across a range of BMIs were recruited. All underwent CMR at 3T to determine LV geometry and function. Group A (75 individuals) underwent $31 \mathrm{P}-$ MRS to assess PCr/ATP. The remaining 70 (group B) - 51 obese (BMI 36.1 \pm 5.3 ), and 19 normal weight (BMI 23.9 \pm 3.9 ) - underwent Triple Repetition time Saturation Transfer 31P-MRS to determine CK kf.

Results Body fat mass and PCr/ATP were negatively correlated in group A $(r=-0.457, p<0.001)$. In group $B$, there were no differences between LV EDV, mass or LVEF between the groups (all $\mathrm{p}<0.05)$. CK forward rate constant $(\mathrm{kf})$ was higher in obese participants than normal weight (CK Kf $0.20 \pm 0.12$ $\mathrm{s}-1$ vs $0.09 \pm 0.07 \mathrm{~s}-1 ; \mathrm{p}=0.001)$. Myocardial CK kf increased in proportion to body fat $(\mathrm{r}=0.283, \mathrm{p}=0.026)$.

Conclusions We studied the effect of obesity on CK kinetics in the human heart, and have demonstrated that, despite a fall in PCr/ATP with increasing body fat, the CK reaction rate is higher in obesity than in normal weight. This may reflect a compensatory increase in CK activity to maintain ATP delivery rates and attempt to preserve cardiac function despite a diminished substrate pool.

\section{P25 HEART FAILURE INCREASES MYOCARDIAL S NITROSYLATION}

${ }^{1} \mathrm{EJ}$ Radcliffe, ${ }^{2} \mathrm{~J}$ Sun, ${ }^{2} \mathrm{~A}$ Aponte, ${ }^{1} \mathrm{DW}$ Eisner, ${ }^{2} \mathrm{E}$ Murphy, ${ }^{1} \mathrm{AW}$ Trafford. ${ }^{1}$ The University of Manchester, UK; ${ }^{2}$ National Heart, Lung and Blood Institute, NIH, USA

10.1136/heartjnl-2018-BSCR.30

The availability of ATP is a limiting factor to cardiac contraction. ATP is predominantly produced by mitochondria via oxidative phosphorylation. Nitric oxide acts as a competitive inhibitor of complex IV and following ischemia, S-nitrosylation has been shown to inhibit complex I activity. Given the established role for nitric oxide in the regulation of oxidative phosphorylation, it is the aim of this study to determine if nitric oxide signalling plays a regulatory role in ATP production in the failing heart.

An ovine tachypaced model of heart failure has been used. 4-6 weeks tachypacing resulted in an increase in left ventricular diameter $(3.10 \pm 0.06 \mathrm{~cm}$ to $4.04 \pm 0.13 \mathrm{~cm}, \mathrm{p}<0.01, \mathrm{n}=5)$ and a reduction in left ventricular contractility $(0.47 \pm 0.01$ to $0.20 \pm 0.03, \mathrm{p}<0.01, \mathrm{n}=5$ ). Following sacrifice, left ventricular, posterior free wall samples were snap frozen and enriched for S-nitrosylation using resin assisted capture. Samples were quantified using mass spectrometry. $232 \pm 18$ discrete proteins were identified as nitrosylated in the control myocardium. This increased to $314 \pm 28.3$ in the heart failure $(p=0.02, n=6)$. Additionally, $79 \%$ of nitrosylated proteins also showed an increase in S-nitrosylation abundance. Several specific sites of S-nitrosylation in heart failure were identified within the electron transport chain; the subunits NDUFS1, SDHA and UQCRH all had a greater than 1 fold increase in nitrosylation in the disease state.

This study is the first to demonstrate a gross increase in the level of myocardial S-NO, and several specific mitochondrial sites of nitrosylation in heart failure. Future work will investigate the functional consequence of nitrosylation at these sites.

\section{P26 INHIBITION OF MITOCHONDRIAL FISSION DECREASED ENDOTHELIAL CELL VON WILLEBRAND FACTOR CONTENT AND GAP JUNCTION COMMUNICATION}

${ }^{1} \mathrm{~S}$ Kaloya, ${ }^{2} \mathrm{~J} \mathrm{McC}$ arron, ${ }^{2} \mathrm{~S}$ Chalmers, ${ }^{1} \mathrm{C}$ McCormick. ${ }^{1}$ Biomedical Engineering Department, University of Strathclyde, Glasgow; ${ }^{2}$ Strathclyde Institute of Pharmacy and Biomedical Sciences (SIPBS), University of Strathclyde, Glasgow

\subsection{6/heartjnl-2018-BSCR.31}

The endothelium is the innermost layer of vascular cells, with a central role in maintaining vascular health. Within endothelial cells, mitochondria play important roles in calcium 
homeostasis, reactive oxygen species production and, to a lesser extent, ATP generation. The balance of mitochondrial fission, fusion and motility is likely to provide fine control of subcellular location and interactions of the organelle; however, the outcome of perturbation of mitochondrial dynamics on endothelial function remains unclear. We sought to address this gap by investigating the effects of mitochondrial fission inhibitor, Mdivi-1, on endothelial cells.

Treatment of cultured endothelial cells with Mdivi-1 (1 or $10 \mu \mathrm{M}, 48$ hour) increased mitochondrial length and branching extent compared to control, consistent with inhibition of fission. Mdivi-1 increased branched, twisted and looped endothelial mitochondrial morphologies, whilst also reducing net mitochondrial speed. No acute toxicity was observed after Mdivi-1 treatment $(10 \mu \mathrm{M}, 48$ hour), however Mdivi-1 did decrease the intracellular content of the glycoprotein von Willebrand Factor (produced, stored and released by endothelial cells to aid thrombosis). Endothelial gap junction communication was also assessed as a function of confluent cells' ability to inter-cellularly transfer the dye, Lucifer yellow; Mdivi-1 decreased dye transfer rates, suggesting reduced intercellular gap junction communication.

In conclusion, Mdivi-1 treatment altered endothelial mitochondrial morphology and dynamics, and also decreased von Willebrand Factor content and gap junction communication, however the mechanistic links remain unclear. Clarification is important, as modulation of mitochondrial dynamics has been proposed as a novel target against the cell proliferation associated with vascular disease.

\section{P27 DEPLETION OF CARDIAC SUCCINATE MEDIATES IMPAIRED HYPOXIA-INDUCIBLE FACTOR 1A SIGNALLING BY LONG CHAIN FATTY ACIDS IN INSULIN RESISTANCE}

${ }^{1} \mathrm{MS}$ Dodd, ${ }^{1} \mathrm{Mdl}$ Sousa Fialho, ${ }^{1} \mathrm{CN}$ Montes Aparico, ${ }^{1} \mathrm{M}$ Kerr, ${ }^{1} \mathrm{KN}$ Timm, ${ }^{2} \mathrm{H}$ Griffin, ${ }^{3}$ JJFP Luiken, ${ }^{3}$ JFC Glatz, ${ }^{1} \mathrm{DJ}$ Tyler, ${ }^{1} \mathrm{LC}$ Heather. 'Department of Physiology, Anatomy and Genetics, University of Oxford, Parks Road, Oxford, UK; ${ }^{2}$ Department of Biochemistry, University of Cambridge, Cambridge, UK; ${ }^{3}$ Department of Molecular Genetics, Cardiovascular Research Institute Maastricht (CARIM), Maastricht University, Maastricht, The Netherlands

10.1136/heartjnl-2018-BSCR.32

Hypoxia-inducible factor (HIF) $1 \alpha$ is activated following myocardial infarction, and is critical for cell survival in hypoxia. In cancer, changes in Krebs cycle intermediates have also been shown to affect HIF1 $\alpha$ stabilisation. We questioned whether abnormal metabolism could prevent HIF $1 \alpha$ activation in diabetes. Type 2 diabetic hearts have decreased HIF $1 \alpha$ protein accumulation in ischemia, which correlated negatively with plasma fatty acid (FA) concentrations and positively with myocardial succinate concentrations. In insulin-resistant cardiomyocytes, HIF signalling and downstream metabolic adaptation was suppressed in hypoxia. Impaired HIF $1 \alpha$ stabilisation was due to increased degradation of the protein in hypoxia, as inhibition of the proteasome or inhibition of the HIF hydroxylases was able to increase HIF1 $\alpha$ in insulin resistance. This was due to abnormal metabolism, as FA (both palmitate and oleate) prevented HIF1 $\alpha$ accumulation in a concentration-dependent manner, which could be reversed by blocking CD36 mediated FA uptake. Succinate promotes HIF stabilisation by inhibiting the HIF hydroxylases, however, FA suppressed succinate accumulation during hypoxia. Increasing succinate concentrations using dimethylfumarate, overrides the FA-mediated inhibition of HIF $1 \alpha$ in a concentration-dependent manner. Pharmacologically inhibiting the HIF hydroxylases promoted HIF $1 \alpha$ accumulation and improved cardiac function following ischemia-reperfusion in diabetic rats. In conclusion, elevated FA in type 2 diabetes prevent HIF $1 \alpha$ accumulation by decreasing succinate concentrations in hypoxia.

\section{P28 IDENTIFICATION OF A SHEAR STRESS RESPONSIVE NOVEL GENE IN THE INTRON OF LAF4}

M Ehteramyan, L Yi, Y Hu, L Zeng. Cardiovascular division, James Black Centre, King's College London, London, UK

\subsection{6/heartjnl-2018-BSCR.33}

A novel gene was recently discovered using microarray gene profiling of shear stress-induced genes in mouse embryonic stem cells (ESCs), which is located within the intron of the transcription factor Laf4 and therefore designated as Laf4 intron resident gene (Laf4ir). Laf4 and Laf4ir genes use different strands for transcription. Two transcript variants have been identified for Laf4ir with three potential opening reading frames (ORFs). In this study, we intend to characterise the transcriptional/translational expression, regulation, and functions of this novel gene. The experiments with qRT-PCR analysis revealed that Laf4ir mRNA was unregulated transiently by shear stress compared to static conditions in mouse differentiated ESCs. Laf4ir was expressed in late stage of mouse embryos but not in the early stages and Laf4ir was differentially expressed in adult mice organs/tissues. Specific antibodies against peptides from the ORFs confirmed the translation of these ORFs in mouse differentiated ESCs in response to shear stress. Immunofluorescence staining with antibody against ORF2 revealed that the ORF2 was exclusively expressed in the intima of aorta in wild type mice. Over-expression of ORF2 significantly increased CD31 expression in mouse ESC-derived Sca1+ cells, indicating the potential role of Laf4ir in endothelial cell differentiation. Interestingly, high level of ORF2 was detected from intima to the adventitia of aorta in ApoE-/- mice and in the adventitia of femoral artery from platinum wire injured mice. Further detailed and concrete investigation is required to characterise the potential functions of Laf4ir gene products, which may provide some new insights into vascular biology.

\section{P29 THE TRANSCRIPTION FACTOR BRN-3B/(POU4F2) REGULATES VASCULAR FUNCTION AND INTEGRITY IN VIVO}

L Mele, $\amalg$ Maskell, VS Budhram-Mahadeo. Medical Molecular Biology Unit, Institute of Cardiovascular Science, University College London, UCL, Rayne Building, London, UK

\subsection{6/heartjnl-2018-BSCR.34}

Rationale Chronic hypertension is a major risk factor for stroke and coronary heart disease. Hypertension is associated with vascular smooth muscle cell (VCMCs) dysfunction, leading to vascular wall calcification and reduced vessel compliance. However, the underlying cellular mechanisms remain unclear. We previously demonstrated that loss of the transcription factor Brn-3b/(POU4F2) results in weight gain and diabetes in mice, known risk factors for hypertension. Herein we present evidences for a direct role of Brn-3b in the maintenance of vascular integrity. 